\title{
Integration of Mobile IPv6 into Mobile Ad-Hoc Network Systems
}

\author{
Monden Kazuya ${ }^{1}$, Satoh Hiroki ${ }^{1}$ Yamamoto Junji ${ }^{2}$, Shomura Yusuke ${ }^{2}$, \\ Shimizu Atsushi ${ }^{1}$, Hayashi Masato ${ }^{1}$, Matsui Susumu ${ }^{1}$, and Yoshizawa Satoshi ${ }^{2}$ \\ ${ }^{1}$ Hitachi, Ltd, Systems Development Laboratory \\ 1099, Aso-ku Ozenji, Kawasaki, 215-0013, Japan \\ \{monden, h-satoh, shimizu, m-hayash, matsui\}@sdl.hitachi.co.jp \\ ${ }^{2}$ Hitachi, Ltd, Central Research Laboratory \\ 1-280, Higashi-Koigakubo, Kokubunji-shi, Tokyo 185-8601, Japan \\ \{junji-y, yusuke-s, yoshi\}@crl.hitachi.co.jp
}

\begin{abstract}
Mobile ad-hoc network (MANET) technology is a wireless network system that may make ubiquitous computing possible. This technology is especially promising for vehicle-to-vehicle and road-to-vehicle communication. However, there are some problems with IP mobility support in MANETs. One technology that provides IP mobility to mobile nodes is Mobile IP. To solve IP mobility problems in MANETs, we examined how Mobile IP technology can be integrated into MANET.
\end{abstract}

\section{Introduction}

Mobile ad-hoc network (MANET) technology, which constructs wireless multihop network autonomously without any infrastructure, is a wireless network system that may make ubiquitous computing possible. In intelligent transport system (ITS) services, a MANET enables vehicle-to-vehicle (V2V) and road-tovehicle $(\mathrm{R} 2 \mathrm{~V})$ communication. In $\mathrm{V} 2 \mathrm{~V}$ communication, vehicles communicate directly with each other without any infrastructure such as base stations. In $\mathrm{R} 2 \mathrm{~V}$ communication, vehicles communicate with road nodes via gateways on the road.

One application of V2V communication is a vehicle control system that prevents rear-end accidents, based on urgent information, such as a sudden braking, sent from a vehicle in front using $\mathrm{V} 2 \mathrm{~V}$ communication. One example of $\mathrm{R} 2 \mathrm{~V}$ communication is vehicle diagnosis using vehicle relationship management [1] technology.

Using V2V communication, if a MANET is composed of vehicles, the route between them is constructed dynamically and a node can communicate with another node outside of its wireless range using multiple hops, without an infrastructure. MANETs are better at dynamic routing than other systems and do not require infrastructures. Using R2V communication, a server node in a network with an infrastructure, like the Internet, can connect with a mobile node via the network. For example, if a MANET is composed of vehicles and a gateway to the network, vehicles can communicate with the server via the 
gateway. When a mobile node moves and connects to a new network via a gateway, it has to acquire a new IP address belonging to the network. However, the node cannot keep communicating with correspondent nodes using the previous IP address. This means that you cannot keep talking with your friends using IP telephony via the Internet while driving. For ubiquitous computing, the talk should continue even if the node that you are using connects to a new network. To accomplish this, Mobile IP [2] 3] has been proposed. Mobile IP is designed to provide IP mobility to mobile nodes over the Internet. Mobile IPv6 [3] is especially promising because it can handle the number of nodes needed in the ITS services that we investigate. Mobile IPv6 has several benefits: First, a mobile node can keep communicating with correspondent nodes using its own unique IP address. Second, the network that the mobile node is connected to can be changed automatically. Therefore, integrating Mobile IPv6 into a MANET is effective in $\mathrm{V} 2 \mathrm{~V}$ and $\mathrm{R} 2 \mathrm{~V}$ communication. However, combining these two technologies presents some problems. One problem is that new IP addresses cannot be acquired using multiple hops. Therefore, we integrated Mobile IPv6 into a MANET to achieve seamless roaming between networks. We will propose our method for combining Mobile Ipv6 and MANETs in Section 4.

\section{MANET and Mobile IPv6}

In this section, we introduce two important technologies: MANET and Mobile IPv6.

\subsection{MANET}

A MANET is a collection of mobile computing devices (network nodes) connected by wireless communication technology. The network has three main features: it is established by instant autonomous networking, it is multi-hopping, and its topology is dynamic. First, nodes discover their neighbors by periodical beaconing, which allows each node to exchange information (node attributes like IP addresses, IDs, information on its neighbors, etc.) with its neighbors to establish connections automatically. Second, if a destination node moves outside radio range of a source node, intermediate nodes help relay data to the destination. Third, when nodes move during communication, each node automatically searches for new neighbors and calculates a new route between a source and its destination, which is called dynamic routing.

Routing protocols fit into two major categories: proactive and reactive. Optimized link state routing (OLSR) protocol 4] is a proactive protocol that each network node uses to periodically exchange topology information with other nodes so that routing tables are ready before nodes send packets. Ad hoc ondemand distance vector (AODV) [5] is a reactive protocol that a source node uses to broadcast route query packets based on requests to send information, and when intermediate nodes receive these packets, they create reverse route entries to the source node. When the destination node receives the query packet, it sends a route reply packet to the source node. This packet gives the order 
of intermediate nodes. Therefore, when a node receives a route reply packet, it makes a routing table entry for the destination node and sends the requested information. OLSR has a shorter delay in sending data than an AODV, but it constantly consuming wireless bandwidth. An AODV has lower routing overhead than OLSR, but taking the time needed to make routes delays transmission. An IETF $^{1}$ working group known as MANET WG $^{2}$ has endeavored to standardize these two protocols.

We use OLSR as our MANET protocol because, in view of time-sensitive applications such as vehicle control systems, a proactive routing protocol that has less delay is better.

\subsection{Mobile IPv6}

Mobile IPv6 is designed to allow nodes to be reached and maintain ongoing connections while changing their locations. Mobile IPv6 provides a function similar to post office forwarding to provide network connectivity to mobile nodes. In Mobile IPv6, each mobile node has a unique IP address, called a home address (HoA). A HoA is permanently assigned to each mobile node within its home subnet prefix on its home link. Each mobile node has a home agent on its home link with which it registers its temporary care-of address (CoA) on the foreign link it is attached to. When a mobile node moves to a foreign network, it receives a router advertisement (RA) packet from a router in the foreign link and therefore, knows it has moved. Its CoA is generated by replacing the node's subnet prefix with the foreign subnet prefix in the RA. The mobile node registers its CoA with its home agent (HA) by sending a binding update message to the HA. The HA replies to the mobile node with a binding acknowledgement message. After finishing this procedure, the HA intercepts packets on the home link destined for the mobile node's home address, encapsulates them, and transmits them to the registered $\mathrm{CoA}$.

\section{Problems in Integration}

This section describes problems in integrating Mobile IPv6 into a MANET.

\subsection{Acquiring Care-of Addresses}

Using Mobile IPv6, a mobile node acquires a foreign subnet prefix by receiving an RA from a router in the network to which it has moved, and then a CoA is generated. Although the RA from the router is transmitted to the all-node multicast address (ff02::1) [6], a multicast packet cannot be forwarded by intermediate nodes because the standardized MANET routing protocols are unicast routing protocols. Therefore, the RA does not reach mobile nodes connected to

\footnotetext{
${ }^{1}$ Internet Engineering Task Force http://www.ietf.org

${ }^{2}$ Mobile Ad-Hoc Networks(manet) http://www.ietf.org/html.charters/manet-charter. html
} 
the router via more than one hop, so that the mobile nodes cannot detect their movements to the foreign link and acquire CoAs. So that they can acquire CoAs, we must develop a method of distributing network prefixes to mobile nodes that are connected to the router with multi-hop wireless links.

\subsection{Routes to CoA in MANETs}

Next, we describe the problem of making routes to CoAs. Using Mobile IPv6, a mobile node that acquires a CoA sends a binding update message to its HA. The HA returns a binding acknowledgement message to the mobile node. To accomplish this, interactive communication must be possible between the mobile node and its HA. The binding update packet must reach the mobile node's HA and the binding acknowledgement packet must reach the mobile node's CoA. This means that a route must be constructed between the mobile node's CoA and HA in the MANET. In OLSR, the route can be constructed by a host and network association message, which is an OLSR control message. The message is used to provide connectivity between the MANET and external networks. However, no standardized way has been developed to make CoAs into routable addresses on MANETs.

We now propose a way to construct routes to mobile nodes' CoAs in a MANET.

\subsection{Redundant Routes to HoAs}

Nodes communicate using their HoAs. Packets are transmitted to HoAs when mobile nodes are attached to links away from their home links, and the packets are encapsulated in packets destined for the nodes' CoAs by the HAs and delivered to the nodes. Even if two nodes in a MANET can communicate with each other without messages passing through their HAs, packets are still transmitted via the HAs. These routes are redundant. Therefore, the delay and the waste of network resources, such as wireless bandwidth, are higher than a system in which communication is done only within the MANET. For example, in a vehicle network, two vehicles connected by multiple hops cannot communicate with each other directly in a MANET without messages passing through the Internet. This means that communication via HAs is not suitable for the time-sensitive applications. Therefore, a communication route that does not pass through the Internet must be constructed.

\subsection{Multiple Wireless Media}

The combination of MANETs and Mobile IPv6 allows mobile nodes to expand the area where the Internet can be accessed via gateways. However, if there is no intermediate node between a mobile node and a gateway, the mobile node cannot communicate via the Internet. This means that we have not yet achieved ubiquitous computing. If a mobile node has multiple wireless interfaces such as a wireless LAN (WLAN) device and a cellular phone and uses them depending on 
which is available, it will communicate using the WLAN where it can communicate with a destination node via a MANET and communicate using the cellular phone where it cannot communicate with a destination node via a MANET. In this case, we assume that MANETs can only be accessed using WLAN interfaces because cell phones cannot directly connect mobile nodes without base stations. Therefore, the problem is how to change between the cellular networks and MANETs.

\section{Solutions to the Problems}

In this section, we propose solutions to the problems we clarified in the previous section. We focus on IPv6-based networks. Therefore, we have to extend OLSR to support IPv6. In IPv4, OLSR control messages are broadcast, but IPv6 has no broadcast address. Control messages are transmitted to the all-node multicast address (ff02::1), in the same way that an RA is advertised. Moreover, we added the following extensions for the integration.

\subsection{Method of Distributing Network Prefixes}

An RA multicast from a router does not reach mobile nodes connected to the router with multiple hops. However, OLSR control packets are flooded to all nodes even if they are connected with multiple hops. This flooding is multipoint relay (MPR) flooding, which is more efficient than traditional flooding. A comparison of traditional flooding and MPR flooding is shown in Figure1, MPRs are special nodes that transmit control messages and are selected by source nodes to reduce retransmission of the messages. Clausen and Jacquet (2003) explain a selection scheme for MPRs [4. We focus on this efficient flooding scheme. To

Traditional flooding

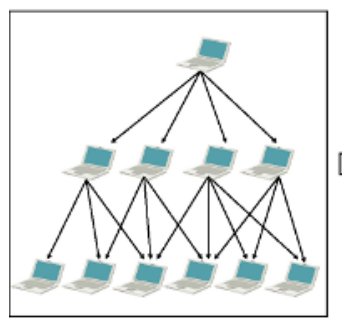

Fig. 1. Comparison of flooding schemes

distribute the foreign subnet prefix to all nodes that can be connected with the router, the packet that stores information on the prefix is transmitted as an OLSR control packet. We call this control packet an RA/OLSR. A mobile node that receives an RA/OLSR can acquire its subnet prefix and generate a CoA. 
The gateway between a MANET and a traditional IP network acts as a router and sends an RA/OLSR. First, a MANET must be established to deliver an RA/OLSR including the foreign subnet prefix. For that, we assign a MANET address, which is used to establish the MANET among mobile nodes.

Now, mobile nodes have three IP addresses.

1. Home address: the permanent address of a mobile node. This address is within the home network of the node. Applications communicate with each other using their home addresses.

2. Care-of address: generated by an RA/OLSR. It is used to communicate with the HA.

3. MANET address: Address used to establish a MANET.

\subsection{Construction of Routes to CoAs}

When a mobile node gets a CoA, it performs a binding update message and receives a binding update acknowledgement. A bidirectional route to the mobile node's HA is necessary to accomplish this procedure. OLSR can provide a route to the mobile node's home link, but a return route must be made to the CoA from HA. The route from the home link to a gateway is constructed in traditional IP networks, so we do not need to construct it. However, a route from the gateway to the mobile node is not provided by MANET routing protocols. In the following, we focus on an OLSR function. OLSR allow mobile nodes to have multiple wireless interfaces that are assigned routable addresses in MANET. This function allows a mobile node to have multiple routable addresses. We have designed a method of assigning MANET addresses and CoAs to the multiple routable addresses. When a mobile node acquires a CoA, we assume that the mobile node got a new wireless interface that was assigned the CoA, or an old interface was reassigned the CoA. Then, the mobile node begins to advertise the $\mathrm{CoA}$ as its multiple routable address. Therefore, the CoA becomes a routable address, and OLSR constructs a route to the CoA in the MANET. This method provides a bidirectional route between a mobile node and its home link.

\subsection{Optimized Route}

In Mobile IPv6, packets sent from a mobile node to another mobile node are transmitted first to the $\mathrm{HA}$ and then are sent to the destination node after the HA changes the destination of the packets to the CoA from the HoA (Figure 2, route $(\mathrm{A}))$. Even if the destination node is a neighbor, this mechanism carries packets to the destination node through a redundant route. Mobile IPv6 has a function that optimizes routes: the source node sends packets directly to the destination node's CoA (Figure 2, route (B)) However, the source node must send a packet to the destination node's HA at least once to know the destination node's CoA. Moreover, the mobile nodes (MNs) are assumed to have two wireless interfaces such as one MANET interface (a WLAN) and one non-MANET interface (a cellular phone), the MNs are assumed to connect with the gateway (GW) 
using the cellular phones, and the two MNs are assumed to connect using the WLAN. The shortest path between the MNs based on the scheme is not route (B) but $\mathrm{MN} 1 \rightarrow \mathrm{GW} \rightarrow \mathrm{MN} 2$ because, in this case, MN's CoA is assigned from the gateway to a cellular network, so it is not a MANET routable address. This optimization scheme is not suitable for time-sensitive communication. Therefore, we propose a new route optimization scheme to integrate Mobile IPv6 into MANET. We focus on a mobile nodes' routing tables. If there is a host route to MN2 in MN1's routing table, MN1 sends packets to MN2 directly because host routes are prioritized over network routes. In reality, the MANET address and $\mathrm{CoA}$ of every $\mathrm{MN}$ are registered in each MN's routing table as host routes. If the $\mathrm{HoA}$ of an $\mathrm{MN}$ can be registered to a routing table as a host route, the route can be optimized. The route can be optimized using a method similar to the construction of the route to the CoA described in the previous paragraph. A HoA becomes a routable address if a mobile node has a virtual interface that is assigned the HoA. After that, OLSR makes a route to the HoA in the MANET so that packets to the HoA in same MANET are transmitted to the corresponding node directly (Figure 2, route (B)).

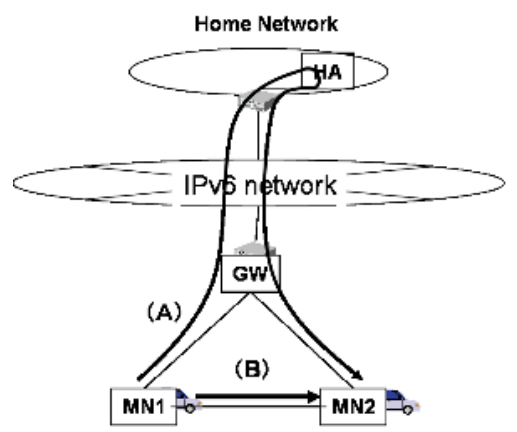

Fig. 2. Redundant route and optimized route

\subsection{Selection Method of Wireless Media}

We focus on mobile nodes that have two wireless devices; one device is a WLAN and the other is a cellular phone. A MANET is established using the WLANs; the cellular network is not a MANET because a cellular phone cannot communicate directly without a base station. We think that a cellular network can complement a MANET. If the mobile node can communicate with its destination node using either interface, it should use the WLAN because of its higher bandwidth and lower communication costs. Available networks, including MANET and cellular networks, can be detected by receiving RAs and RA/OLSRs. Therefore, the interface used to communicate is switched based on the reception or time-out of RA/OLSRs. 


\section{Experimentation}

We implemented the solutions developed in Section 4 to construct the system shown in Figure 3 .

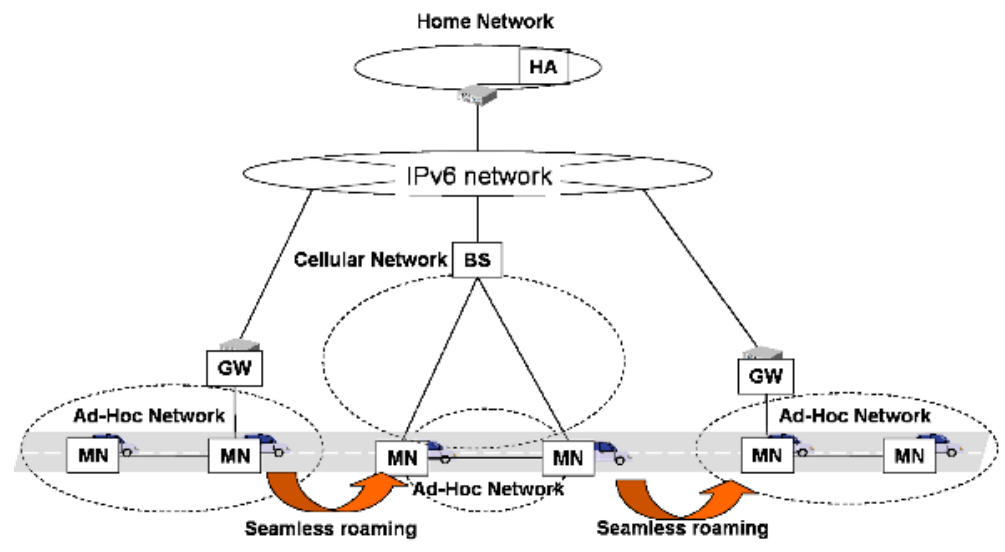

Fig. 3. Structure of network

\subsection{Structure of Experimental System}

Each mobile node has two communication interfaces: a wireless LAN interface and a cellular phone. Extended OLSR is installed on each MN. MANETs use only wireless LAN. Gateways connect the MANETs and an IPv6 wired network. The gateways have two interfaces: wireless LAN and wired LAN. The gateways periodically send RA/OLSRs as routers. The MNs seamlessly communicate with destination nodes using their wireless LANs and cellular phones. A HA is in each MN's home network. A MN's home network, gateways, and base stations (BSs) of cellular networks are routable with each other. The MN communicates seamlessly by switching between MANETs and cellular networks.

\subsection{Applications}

We used video streaming between the mobile nodes as an example of a service. One application of the streaming is the Video Conferencing Tool (VIC) ${ }^{3}$. We developed software to monitor nodes' available routes and the positions of the nodes based on global positioning system (GPS) information.

\subsection{Experimental Conditions}

We prepared two laptops as MNs and put them in vehicles (Figure 4). Each MN's wireless LAN device was IEEE802.11b. We attached external antennas to wireless LAN devices and GPS device on the roof, as shown in Figure [5] MN1 had a camera for video streaming. The vehicles went around a circuit of about

\footnotetext{
${ }^{3}$ http://www-nrg.ee.lbl.gov/vic/
} 


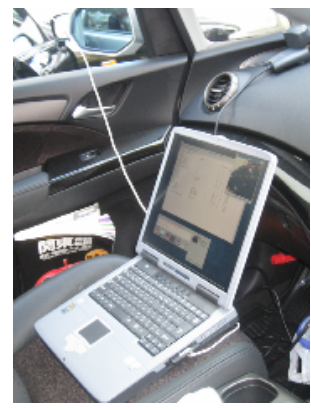

Fig. 4. Mobile node in a vehicle

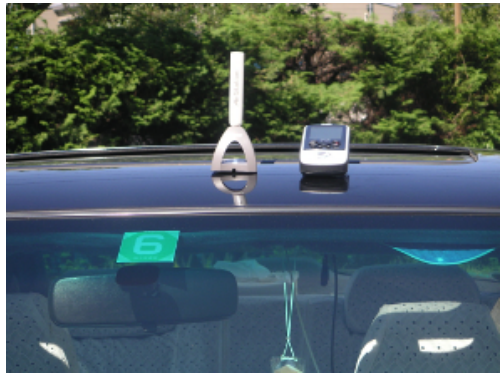

Fig. 5. Left:external antenna Right: GPS device

$2 \mathrm{~km}$ (Figure 6) at $40 \mathrm{~km} / \mathrm{h}$. Two gateways (GW1 and GW2 in Figure 6) were located near the circuit. The gateways were connected to the IPv6 Internet using asymmetric digital subscriber lines (ADSL). The routes available to each node were checked on MN2 using the monitoring software we developed. The quality of the communication was tested by examining the quality of a video stream of VIC transmitted from MN1. The feasibility of the communication was done by available routes and GPS information transmitted from other nodes including GWs. The vehicles went around the track twice. On first lap, the two vehicles drove together. On second lap, MN2 stopped near GW1, and MN1 went around alone. We tested the following cases.

Case 1. A MN connected to a gateway between a MANET and the Internet by multiple-hops was sent an RA/OLSR. We checked that the MN received it.

Case 2. A MN generated a CoA and sent a binding update. We checked to see if a binding acknowledgement was received.

Case 3. The path between MN1 and MN2 was tested to see if it was the shortest.

Case 4. We checked that MNs switched communication interfaces to the Internet as available and continued communicating with destination nodes.

\section{Results}

The following figures are screen shots captured on MN2. First, we will explain the screen elements. In Figure 7 the upper left window is the software that monitors the node's available routes and its position. Each node is a box, and MNs are cars. The node labeled "Relay" was a fixed relay node, placed where it is because GW1 was not close enough to the circuit. The lines show available routes. Lines between GWs and the Internet show that two gateways are connected via the Internet using ADSL. Lines between cars and the Internet show a direct connection to the Internet using a cellular network or a connection between MNs in a MANET. Every node sent information, such available routes and its routable address, to MN2's HoA. The upper right window shows video streaming from MN1. Under the left window is a message application, similar to a chat, 


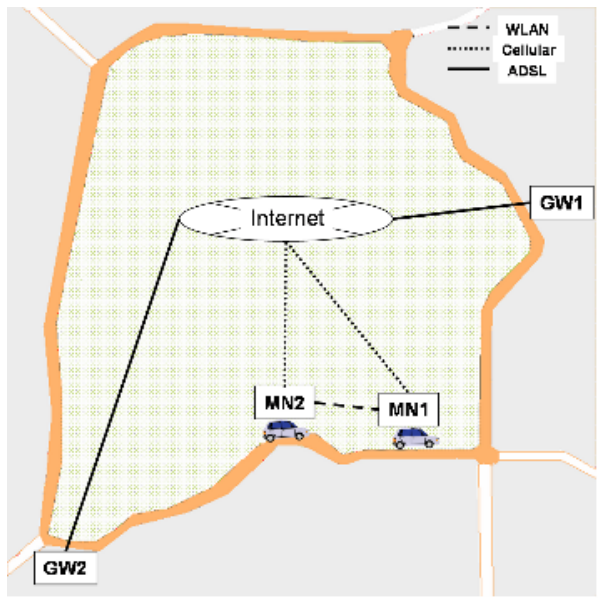

Fig. 6. Experimental circuit

and it shows urgent messages from MN1. Under the right window is a control terminal. Blue and red balls show routes between MN1 and MN2; blue balls show routes from MN2 to MN1, and red balls show return routes from MN1.

\subsection{Case 1 and Case 2}

MN2 was connected to the Internet by multiple hops, generated its CoA, and constructed a route to its CoA. We know this because the receipt of GW1's and Relay's routing information packets by MN2 was displayed in the monitor window (Figure 8). An RA/OLSR was relayed by MN1. MN2 generated its CoA, sent a binding update, and received a binding acknowledgement. This means that the tests of cases 1 and 2 were successful.

\subsection{Case 3}

MNs chose optimized routes even if they could choose other routes via GW1 and cellular networks, as shown in Figures 7 and 9] Therefore, our optimizing method works well.

\subsection{Case 4}

The MNs connected to the Internet via a MANET, and the route between them was in the MANET, as shown in Figure 9. Figure 10 shows a later time than Figure 9. MN2 stopped near GW2. MN1 kept driving and changed the interface it used to access the Internet from its wireless LAN to its cellular phone. The media was changed because MN1 didn't receive an RA/OLSR from GW1. After the moment shown in Figure 10, MN1 connected to GW2, as shown in Figure 11. This figure shows that MN1 changed the interface to its wireless LAN after receiving an RA/OLSR, so MN1 and MN2 continued communicating. These 


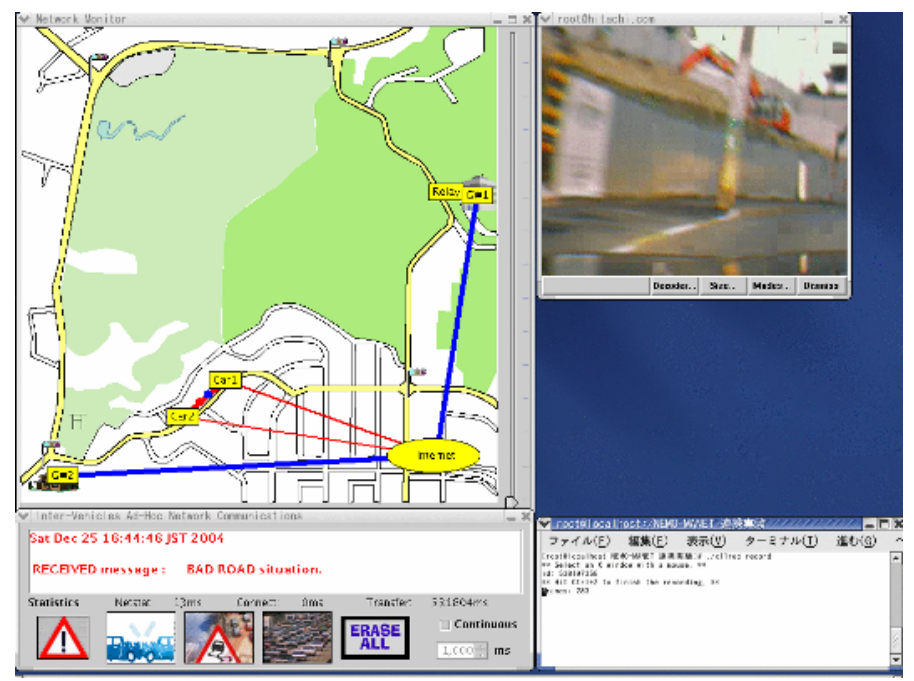

Fig. 7. MN1 $\rightarrow$ MANET $\rightarrow$ MN2

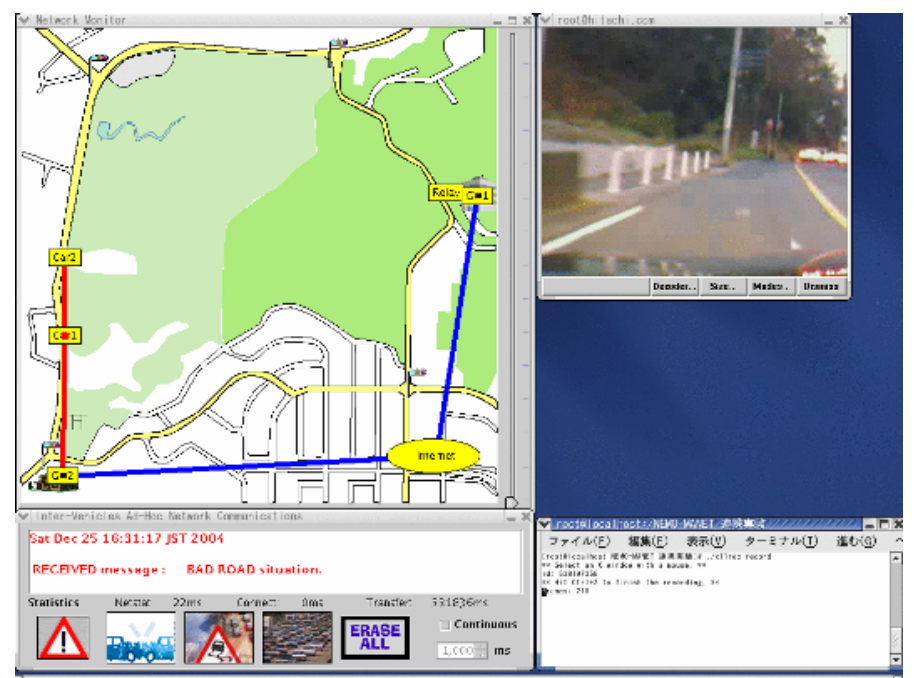

Fig. 8. Multi-hop route to Internet via gateway

three figures ( 9, 10, and 11) show that MN1 changed the interface while driving, but the communication continued. This means that our media selection method allow MN1 to seamlessly change its interface. However, at the time that the network was switched, the communication was cut off. We believe the reason is that the available networks had already changed to only the cellular network by the time the RA/OLSR timed out. 


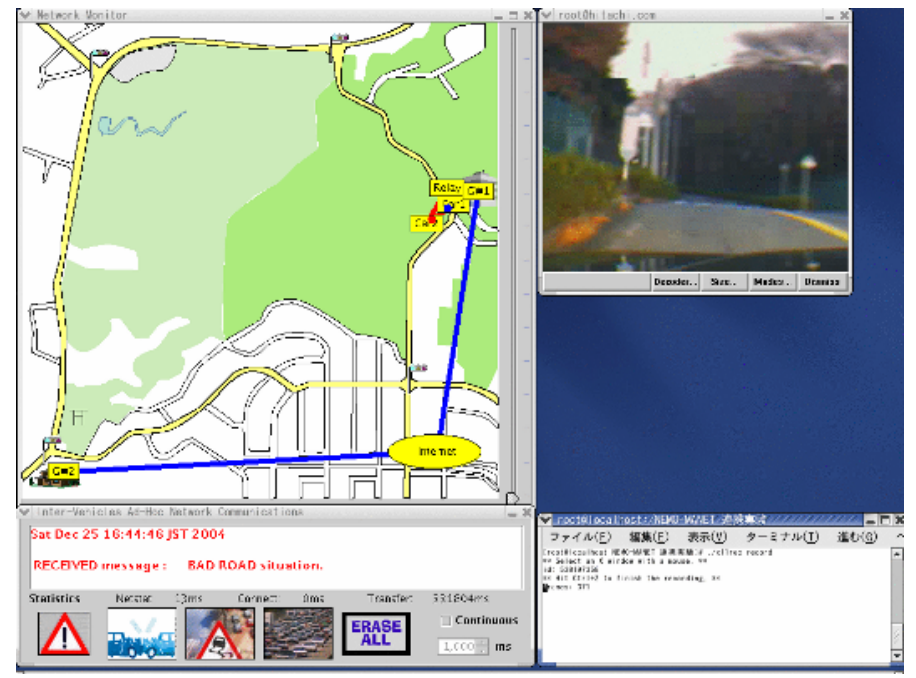

Fig. 9. $\mathrm{MN} 1 \rightarrow \mathrm{MANET} \rightarrow \mathrm{MN} 2$

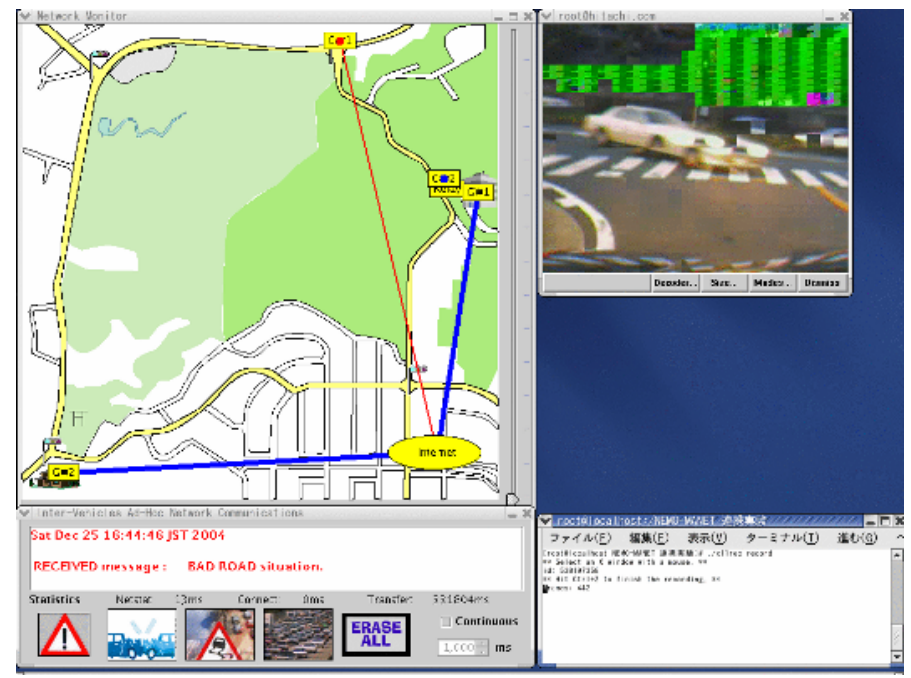

Fig. 10. MN1 $\rightarrow$ Cellular $\rightarrow$ Internet $\rightarrow$ MANET $\rightarrow$ MN2

\subsection{Communication Quality}

The picture from MN1 in Figure 10 has block noise, but the other screen captures have no block noise. Previous routes from MN1 to MN2 passed through a cellular network. Therefore, one reason for the difference of video quality is that the cellular network had a narrower bandwidth than the wireless LAN. MANETs 


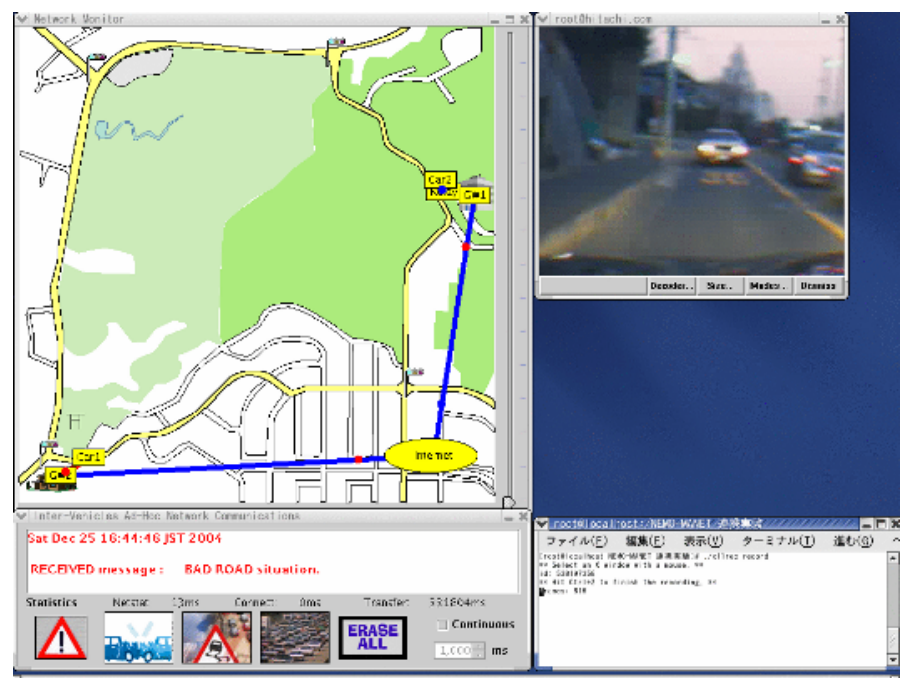

Fig. 11. MN1 $\rightarrow$ MANET $\rightarrow$ Internet $\rightarrow$ MANET $\rightarrow$ MN2

enabled the broadband area to be extended, Mobile IPv6 provided IP mobility to MNs, and the cellular network complemented the MANETs.

\section{Conclusion}

We studied the integration of Mobile IPv6 into a mobile ad-hoc network system and came to the following conclusions.

1. Mobile nodes in a MANET can be connected to the Internet with foreign subnet prefixes that a gateway between the Internet and the MANET sends, included in an RA/OLSR.

2. Advertising a $\mathrm{CoA}$ as a multiple routable address allows mobile nodes to create bidirectional routes to their home links.

3. Routes can be optimized by constructing host routes to destination nodes' HoAs in a MANET.

4. Detection of MANET availability based on receiving and time-out RA/ OLSRs allowed MN1 to change its interface.

5. Integration of Mobile IPv6 into a MANET can expand the range of wireless LAN and enables mobile nodes to communicate seamlessly when they move to new networks.

This integration system can optimize routes, reduce delay, and lower communication costs according to the destination of a packet. This system is a necessary network for ubiquitous computing. The system can be applied not only to ITS systems, but also to other wireless communication systems. Next, we will study the possibility of reducing the period in which communication is impossible by changing the network and extending Mobile IPv6 to network mobility [7]. 


\section{References}

1. Aizono, T., Endo, Y., Otsuji, S., Yamane, K., Shimura, A.: Hitachi groups initiatives regarding trends in vehicle information systems. HITACHI REVIEW 53 (2004) 229-235

2. Perkins, C.: RFC 3220: IP Mobility Support for IPv4 (2002) http://www.ietf. org/rfc/rfc3344.txt.

3. Johnson, D., Perkins, C., Arkko, J.: RFC 3775: Mobility Support in IPv6 (2004) http://www.ietf.org/rfc/rfc3775.txt.

4. Clausen, T., Jacquet, P.: RFC 3626: Optimized Link State Routing Protocol (OLSR) (2003) http://www.ietf.org/rfc/rfc3626.txt.

5. Perkins, C., Belding-Royer, E., Das, S.: RFC 3561: Ad hoc On-Demand Distance Vector (AODV) Routing (2003) http://www.ietf.org/rfc/rfc3561.txt.

6. Narten, T., Nordmark, E., Simpson, W.: RFC 2461: Neighbor Discovery for IP Version 6 (IPv6) (1998) http://www.ietf.org/rfc/rfc2461.txt.

7. Devarapalli, V., Wakikawa, R., Petrescu, A., Thubert, P.: Internet-draft: Network Mobility (NEMO) Basic Support Protocol (2004) http://www.ietf.org/internetdrafts/draft-ietf-nemo-basic-support-03.txt. 